\title{
Estrutura de prática e idade no processo adaptativo da aprendizagem de uma tarefa de "timing" coincidente
}

\section{Resumo}

0 objetivo desse estudo foi investigar os efeitos de diferentes estruturas de prática no processo adaptativo da aprendizagem de uma tarefa de "timing" coincidente em função da idade. Crianças $(n=40)$, adultos $(n=47)$ e idosos $(n=57)$ foram distribuidos em grupos de prática constante, aleatória, constantealeatória e aleatória-constante. A tarefa consistiu consistia em tocar certos alvos de forma sequencial em integração a um estímulo visual. 0 delineamento envolveu duas fases de aprendizagem: estabilização e adaptação. Os dados foram analisados em relação aos erros absoluto, variável, constante e de execução. Os resultados mostraram que o processo adaptativo na aprendizagem de crianças, adultos e idosos de uma tarefa de "timing" coincidente foi mais beneficiado pela prática constante-aleatória.

UnItermos: Estrutura de prática; Idade; Tarefa de "timing" coincidente; Processo adaptativo; Aprendizagem motora.

\section{Introdução}

Dentre as diversas abordagens de pesquisa no campo da Aprendizagem Motora, nos últimos anos os efeitos de diferentes estruturas de prática na aprendizagem de habilidades motoras têm sido investigados sob uma denominada de processo adaptativo de aprendizagem motora (CORRÊA \& TANI, 2005). Trata-se de uma abordagem em que a aprendizagem é explicada por meio de duas fases (CHOSHI, 2000; TANI, 2005): estabilização e adaptação. A fase de estabilização é aquela na qual ocorre a busca pela estabilidade funcional através de feedback negativo. Ela implica na padronização espacial e temporal da habilidade motora. Assim, os movimentos que no início são imprecisos e inconsistentes, gradualmente vão se tornando padronizados, coordenados e precisos.

Já a fase de adaptação diz respeito àquela na qual ocorre quebra da estabilidade (perturbação) e posterior busca por nova estabilidade, ou seja, ela refere-se à adaptação às novas situações ou tarefas motoras com base em habilidades já adquiridas. Quando a perturbação é pequena, dentro da previsibilidade do sistema, a adaptação pode ocorrer pela própria flexibilidade inerente à estrutura, ou seja, pela mudança de parâmetros da habilidade. Porém, há perturbaçôes em que não há condições de se adaptar, pois estão fora da previsibilidade do sistema e exigem uma reorganização na estrutura da habilidade, alcançada num nível superior de complexidade. E há, ainda, um terceiro tipo de adaptação, quando uma reorganização de estrutura não é suficiente, ocorre, então, um processo de auto-organização, com o surgimento de estruturas completamente novas (TANI, 2005).

Os estudos sobre os efeitos da estrutura de prática no processo adaptativo em aprendizagem motora têm manipulado as seguintes estruturas de prática: constante, variada aleatória, constante seguida de variada aleatória, variada aleatória seguida de constante e, constante seguida de variada por blocos (BARROS, 2006; Corrêa, 2001, 2007; Corrêa, Barros, Massigli, Gonçalves, Tani, 2007; Corrêa, Benda, Meira Júnior \& Tani, 2003; Corrêa, Benda \& Tani, 2001; Corrêa, GonçalVes, Barros, Massigli, 2006; Fialho, 2007; Massigli, Gonçalves, BarRos, SOUZa Júnior, Corrêa, 2004; Paroli, 2004; Pinheiro \& Corrêa, 2007; Silva, Araújo, Tani \& Corrêa, 2009; Tertuliano, 2007; Walter, 2007). 
De modo geral, os resultados têm mostrado que a prática constante seguida pela prática aleatória promove uma melhor adaptação a novas situações. Uma explicação para isso é que num primeiro momento, a prática constante possibilita a formação do padrão de interação entre os componentes do sistema, ou seja, a formação da estrutura da habilidade; e a posterior inserção da prática aleatória possibilita um aumento do leque de interações, ou seja, promove a diversificação da habilidade (CORRÊA, 2001; CORRÊA \& TANI, 2005).

Três aspectos dessas pesquisas chamam a atenção: a) há apenas dois estudos realizados com adultos, nos quais apenas a prática constante seguida pela prática variada aleatória foi testada num estudo (FialHo, 2007), e a prática constante seguida pela prática variada por blocos foi considerada melhor do que a prática constante seguida pela prática variada aleatória noutro estudo (PAROLI, 2004); b) não há estudos realizados com idosos; e, por fim, c) nenhum estudo investigou a aprendizagem de indivíduos de diferentes idades em um único delineamento.

Além desses aspectos, um outro dado interessante dessas pesquisas é que a maior parte delas utilizou tarefas de "timing" coincidente como tarefa de aprendizagem. Trata-se de uma tarefa amplamente utilizada na área de Aprendizagem Motora em virtude de requerer do aprendiz desenvolvimento cognitivo e maturação de estruturas visuais e motoras, já que ela depende de outras capacidades, como percepção visual e processamento de informações (Bard, Fleury \& Gagnon, 1990).

BARREIros (1992) salienta a necessidade de se verificar o desenvolvimento de capacidades inerentes à tarefa experimental. Portanto, apontar e compreender as características dos sujeitos em cada fase de desenvolvimento na tarefa a ser utilizada parece ser fundamental. Segundo Bard, Fleury e Gagnon (1990), alguns estudos mostram que essa capacidade de "timing" coincidente pode ser observada em bebês de quatro meses de idade, porém, o seu desenvolvimento ideal ocorre entre os 10 e 12 anos de idade. Com relação ao processamento de informações, que se refere ao processo de controle e de escolha de estratégias, as mudanças dessa capacidade são as principais responsáveis pela melhora ou piora na desempenho em tarefas de "timing" coincidente associadas à idade.

Em síntese, a literatura tem sugerido sugere a existência de relação entre idade e o efeito de diferentes estruturas de prática (BARREIros, 1994; Douvis, 2005; Pollock \& Lee, 1997; Shea, Park \& BraDEN, 2006), sendo que essas pesquisas têm apontado um efeito positivo da prática aleatória para crianças, enquanto que o mesmo efeito não tem sido encontrado em adultos. Além disso, verifica-se, também, relação entre idade e tarefas de "timing" coincidente (Corrêa, Oliveira, Oliveira, Freudenheim, Paroli, Ugrinowitsch, Meira Júnior, Marinovic, SiMONI \& TANi, 2005; FerRaZ, 1993; Fleury \& Bard, 1985; Santos, Corrêa \& Freudenheim, 2003; SANTOS \& TANI, 1995), já que crianças apresentam bom desempenho nessas tarefas com idades entre 10 e 12 anos, as mudanças ao longo da fase adultas não alteram o seu desempenho e, com relação aos idosos, ocorre uma queda de desempenho nesse tipo de tarefa já a partir dos 60 anos de idade. Porém, que esses três aspectos, estrutura de prática, idade e tarefa de "timing" coincidente, ainda não foram investigados sob um mesmo delineamento metodológico, numa perspectiva de processo adaptativo de aprendizagem motora.

A partir desses destaques, pergunta-se: quais seriam os efeitos de diferentes estruturas de prática no processo adaptativo da aprendizagem de uma tarefa de "timing" coincidente em indivíduos de diferentes idades? O objetivo do presente estudo foi investigar os efeitos de diferentes estruturas da prática no processo adaptativo da aprendizagem de uma tarefa de "timing" coincidente em função da idade.

\section{Métodos}

\section{Amostra}

A amostra foi composta da seguinte forma: 40 crianças (10-12 anos) com média de idade de 10,9 $( \pm 0,8)$ anos; 47 adultos (30-40 anos) com média de idade de 34,4 $( \pm 3,9)$ anos; 57 idosos (60-70 anos) com média de idade de $64,5( \pm 3,8)$ anos. A participação no experimento foi condicionada ao consentimento livre e esclarecido assinado por um responsável da criança, ou pelo próprio indivíduo (adultos e idosos). O presente estudo foi aprovado pelo Comitê de Ética em Pesquisa da Escola de Educação Física e Esporte da Universidade de São Paulo (protocolo no 2008/31) 


\section{Tarefa e instrumento}

A tarefa foi de "timing" coincidente que consistiu em tocar cinco alvos numa ordem pré-estabelecida, integrado ao estímulo visual. Conforme ilustra a FIGURA 1, para a realização da pesquisa foi utilizado o aparelho de "timing" coincidente em tarefas complexas (CORRÊA \& TANI, 2004).

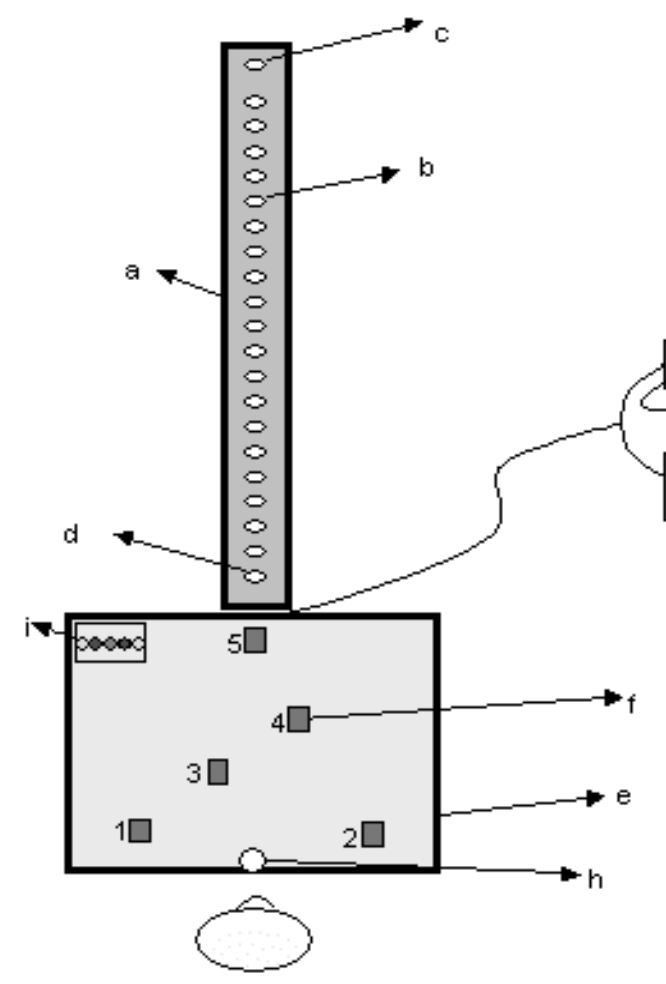

$\mathrm{a}=$ canalet $\mathrm{b}=$ diodos (leds); $c=$ diodo de alerta; $\mathrm{d}=$ diodo alvo; $\mathrm{e}=$ mesa;

$\mathrm{f}=$ sensores de 1 a 5 ; $\mathrm{g}=$ computador; $\mathrm{h}=$ local de posicionamento da mão de execução; $\mathrm{i}$ = "feedback" visual.

FIGURA 1 - Ilustração do aparelho de "timing” coincidente em tarefas complexas (CORRÊA \& TANI, 2004).

\section{Delineamento e procedimentos}

O delineamento foi semelhante àquele utilizado por CoRrêA (2001). As crianças, os adultos e os idosos foram separados aleatoriamente em quatro grupos de prática: constante $(\mathrm{C})$, aleatória $(\mathrm{A})$, constante-aleatória (C-A) e aleatória-constante (AC), formando um total de 12 grupos experimentais.

O experimento constou com duas fases: 1) fase de estabilização, na qual os sujeitos realizaram 72 tentativas; 2) fase de adaptação, na qual foram executadas, em todos os grupos e em todas as idades, 36 novas tentativas, com uma tarefa diferente daquela praticada na fase anterior. Especificamente, houve modificação na sequência de movimentos e na velocidade do estímulo visual.

$\mathrm{Na}$ fase de estabilização, os sujeitos do grupo de prática constante executaram as tentativas numa única velocidade do estímulo visual $(\mathrm{V} 1=142,2$ $\mathrm{cm} / \mathrm{s}$ ) sequência de toques $[\mathrm{S} 1=1-2-4-3-5$ (FIGURA 1)]. Os sujeitos do grupo de prática aleatória executaram as tentativas variando-as em três velocidades do estímulo visual $(\mathrm{V} 1=142,2 \mathrm{~cm} / \mathrm{s}$,
$\mathrm{V} 2=165,7 \mathrm{~cm} / \mathrm{s}$, e V3 $=124,5 \mathrm{~cm} / \mathrm{s}$ ) e em três sequências de toques $(S 1=1-2-4-3-5 ; S 2=1-3-2-4-5$; $S 3=1-4-2-3-5)$ combinadas entre si aleatoriamente, mas com a mesma quantidade de tentativas para todas as possibilidades de combinação. No grupo de prática constante-aleatória, os sujeitos executaram a primeira metade das tentativas da mesma forma daqueles do grupo de prática constante $(\mathrm{V} 1=142,2$ $\mathrm{cm} / \mathrm{s}$ e $\mathrm{S} 1=1-2-4-3-5)$, e as tentativas posteriores semelhantes àqueles do grupo de prática aleatória $(\mathrm{V} 1=142,2 \mathrm{~cm} / \mathrm{s}, \mathrm{V} 2=165,7 \mathrm{~cm} / \mathrm{s}, \mathrm{e} \mathrm{V} 3=124,5$ $\mathrm{cm} / \mathrm{s}$ e $\mathrm{S} 1=1-2-4-3-5 ; \mathrm{S} 2=1-3-2-4-5 ; \mathrm{S} 3=1-4-2-$ 3-5). E, por último, os sujeitos do grupo de prática aleatória-constante executaram a primeira metade de tentativas conforme os sujeitos do grupo de prática aleatória e, a segunda metade, de acordo com os sujeitos da prática constante.

$\mathrm{Na}$ fase de adaptação, todos os grupos executaram as tentativas em uma mesma velocidade do estímulo visual (V4 = 104,9 cm/s) e única sequência de toques (S4 = 1-4-3-2-5), diferente daquelas praticadas na fase de estabilização. Nessa fase não foi fornecido o "feedback" aos sujeitos. 


\section{Tratamento de dados}

Os resultados foram analisados com relação às seguintes medidas de desempenho: erro absoluto (precisão), erro variável (consistência) e erro constante (direção do desempenho). Os dados foram analisados em blocos de nove tentativas, formando, no total, oito blocos na fase de estabilização e quatro blocos na fase de adaptação.
Com relação às análises inferenciais, foram realizados, na fase de estabilização, ANOVAs "one-way" para verificar diferenças entre os oito blocos de tentativas. $\mathrm{Na}$ fase de adaptação realizou-se a ANOVA three-way (3 idades x 4 estruturas de prática x 5 blocos de tentativas) com medidas repetidas no fator blocos de tentativas. $\mathrm{O}$ teste de Tukey foi utilizado para localizar a diferenças encontradas.

\section{Resultados}

\section{Erro absoluto}

\section{Fase de estabilização}

No que diz respeito às crianças, conforme se pode observar na FIGURA 2, todos os grupos melhoraram o desempenho ao longo da fase de aquisição. Esse comportamento foi confirmado pelos testes estatísticos: prática constante $[\mathrm{F}(7 ; 63)=11,9, \mathrm{p}<$ $0,01]$, prática constante-aleatória $[\mathrm{F}(7 ; 63)=4,7, \mathrm{p}$ $<0,01]$, prática aleatória $[\mathrm{F}(7 ; 63)=9,4, \mathrm{p}<0,01]$ e prática aleatória-constante $[\mathrm{F}(7 ; 63)=7,2, \mathrm{p}<0,01]$.

No que concerne aos adultos, ao observar a FIGURA 2 verifica-se que, assim como ocorreu com as crianças, todos os grupos melhoraram o desempenho ao longo da fase de estabilização, o que também foi confirmado pelos testes estatísticos: prática constante $[\mathrm{F}(7 ; 77)=$ 3,57, $\mathrm{p}<0,01]$, prática constante-aleatória $[\mathrm{F}(7 ; 70)=$ $3,7, \mathrm{p}<0,01]$, prática aleatória $[\mathrm{F}(7 ; 77)=3,9, \mathrm{p}<0,01]$ e prática aleatória-constante $[\mathrm{F}(7 ; 77)=5,9, \mathrm{p}<0,01]$.

$\mathrm{E}$, referente aos idosos observa-se que todos os grupos diminuíram o erro absoluto ao longo da fase de estabilização (FIGURA 2). As ANOVAS “one-way” encontraram os seguintes resultados que confirmaram as citadas observaçôes: prática constante $[\mathrm{F}(7 ; 91)=$ $11,3, \mathrm{p}<0,01]$, prática constante-aleatória $[\mathrm{F}(7 ; 91)=$ $3,7, \mathrm{p}<0,01]$, prática aleatória $[\mathrm{F}(7 ; 98)=7,3, \mathrm{p}<0,01]$ e prática aleatória-constante $[\mathrm{F}(7 ; 91)=11,0, \mathrm{p}<0,01]$.

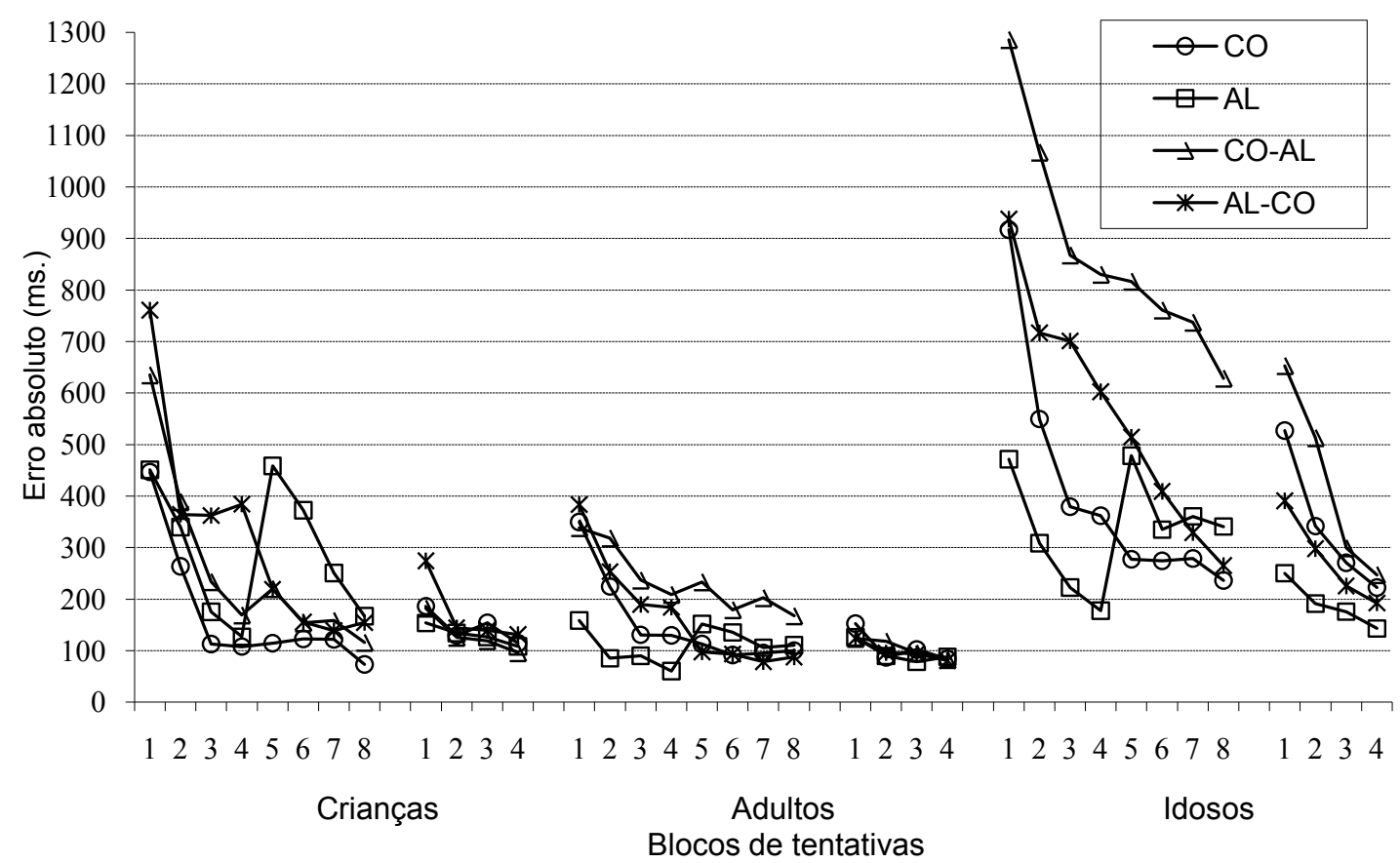

FIGURA 2 - Médias do erro absoluto, em milissegundos, em blocos de nove tentativas, dos quatro grupos de prática constante $(\mathrm{CO})$, constante-aleatória (CO-AL), aleatória (AL) e aleatória-constante (AL-CO), de crianças, adultos e idosos, das fases de aquisição (1 a 8) e adaptação (1 a 4). 
Fase de adaptação

A análise de variância a três fatores para a fase de adaptação apontou diferenças para os fatores idade $[\mathrm{F}(2 ; 132)=10,54, \mathrm{p}<0,01]$ e blocos de tentativas $[\mathrm{F}(4 ; 528)=14,25, \mathrm{p}<0,01]$. Encontrou, também, interação entre idade e blocos de tentativas $[\mathrm{F}(8$; $528)=4,67, \mathrm{p}<0,01]$ e, entre estrutura de prática e blocos de tentativas $[\mathrm{F}(12 ; 528)=2,02, \mathrm{p}=0,02]$. Não foram detectadas diferenças no fator estrutura de prática $[\mathrm{F}(3 ; 132)=0,68, \mathrm{p}>0,5]$; interação entre idade e estrutura de prática $[\mathrm{F}(6,132)=0,77$, $\mathrm{p}>0,6]$; e interação entre idade, estrutura de prática e blocos de tentativas $[\mathrm{F}(24 ; 528)=1,08, \mathrm{p}>0,4]$.

Esses resultados mostraram que: a) crianças e adultos tiveram melhor desempenho do que idosos; b) os grupos foram perturbados com a modificação da tarefa; c) os idosos melhoraram o desempenho após perturbação; d) a perturbação foi mais evidente para os grupos de prática constante; e) os grupos de prática aleatória foram aqueles com pior desempenho; e, f) no início da fase de adaptação os grupos de prática constante-aleatória tiveram melhores desempenhos do que os grupos de prática aleatória.

\section{Erro variável}

\section{Fase de aquisição}

Com relação às crianças, conforme se pode observar na FIGURA 3 todos os grupos diminuíram a variabilidade do desempenho ao longo da fase de estabilização, o que foi confirmado pelos testes estatísticos: prática constante $[\mathrm{F}(6 ; 63)=8,6, \mathrm{p}<$ $0,01]$, constante-aleatória $[\mathrm{F}(6 ; 63)=5,5, \mathrm{p}<0,01]$, prática aleatória $[\mathrm{F}(6 ; 63)=6,2, \mathrm{p}<0,01]$ e prática aleatória-constante $[\mathrm{F}(6 ; 63)=5,2, \mathrm{p}<0,01]$.

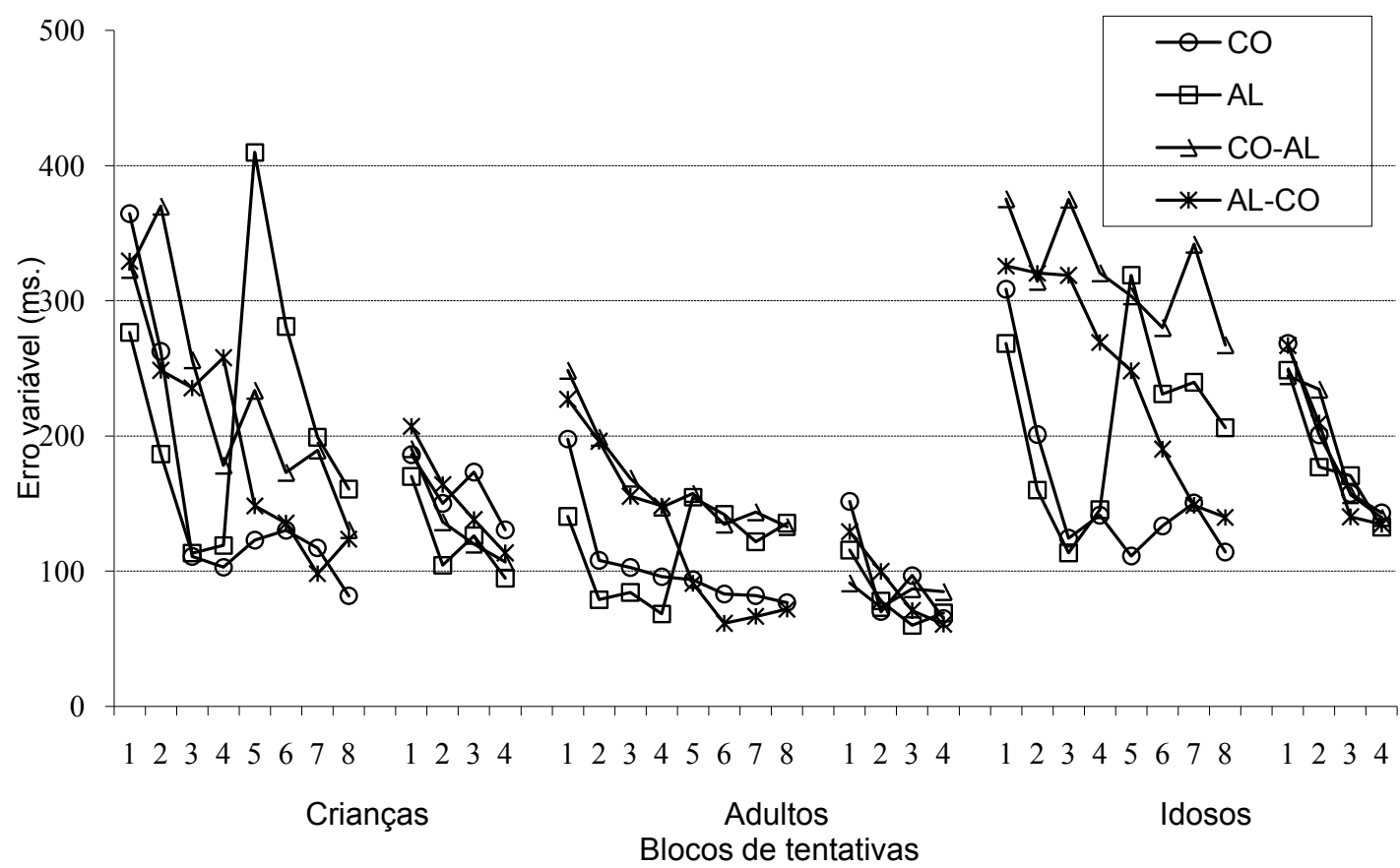

FIGURA 3 - Médias do erro variável em milissegundos, dos grupos de prática constante (CO), constante-aleatória (CO-AL), aleatória (AL) e aleatória-constante (AL-CO), das crianças, dos adultos e dos idosos, nos oito blocos de tentativas da fase de aquisição (1 a 8) e nos quatro blocos de tentativas da fase de adaptação (1 a 4).

Em relação aos adultos, verifica-se que, assim como ocorreu com as crianças, todos os grupos diminuíram o erro variável ao longo da fase de estabilização: prática constante $[\mathrm{F}(7 ; 77)=4,55, \mathrm{p}<0,01]$, prática constantealeatória $[\mathrm{F}(7 ; 70)=5,06, \mathrm{p}<0,01]$, prática aleatória $[\mathrm{F}(7 ; 77)=3,19, \mathrm{p}<0,01]$ e prática aleatória-constante.

$\mathrm{E}$, no tocante aos idosos, conforme a FIGURA 3 todos os grupos diminuíram o erro variável ao longo da fase de estabilização. Entretanto, isso foi parcialmente confirmado pelos testes estatísticos: prática constante $[\mathrm{F}(7 ; 91)=7,81, \mathrm{p}<0,01]$, prática constante-aleatória $[\mathrm{F}(7 ; 91)=7,30, \mathrm{p}<0,01]$, prática aleatória-constante $[\mathrm{F}(7 ; 91)=5,44, \mathrm{p}<$ $0,01]$. Com relação à prática aleatória, a ANOVA "one-way" não encontrou diferença significante $[\mathrm{F}(7 ; 98)=1,02, \mathrm{p}>0,05]$. 
Fase de adaptação

A análise de variância a três fatores apontou diferenças significantes para o fator idade $[\mathrm{F}(2 ; 132)=$ $20,60, \mathrm{p}<0,00]$, para o fator blocos de tentativas [F(4; $528)=26,11, \mathrm{p}<0,00]$, para a interação entre idade e blocos de tentativas $[\mathrm{F}(8 ; 528)=3,11, \mathrm{p}<0,00]$ e, para a interação entre estrutura de prática e blocos de tentativas $[\mathrm{F}(12 ; 528)=3,98, \mathrm{p}=0,00]$. Não foram detectadas diferenças significantes para o fator estrutura de prática $[\mathrm{F}(3 ; 132)=0,12, \mathrm{p}>0,9]$; para a interação entre idade e estrutura de prática $[\mathrm{F}(6 ; 132)=0,23, \mathrm{p}>$ $0,9]$; e para a interação entre idade, estrutura de prática e blocos de tentativas $[\mathrm{F}(24 ; 528)=0,71, \mathrm{p}>0,8]$.

Nessa fase, adultos mostraram mais consistência do que idosos e crianças e, esses últimos foram mais consistentes do que idosos. Os resultados mostraram, também, que todos os grupos foram perturbados com a modificação na tarefa, mas que o desempenho foi retomado no decorrer da fase.

\section{Erro constante}

\section{Fase de estabilização}

No que se refere às crianças, conforme se pode observar na FIGURA 4, todos os grupos diminuíram erro constante, em direção à coincidência, ao longo da fase de estabilização. Essa observação foi confirmada pelas análises estatísticas: prática constante $[\mathrm{F}(7 ; 63)=10,87, \mathrm{p}<0,01]$, prática constante-aleatória $[\mathrm{F}(7 ; 63)=3,62, \mathrm{p}<0,01]$, prática aleatória $[\mathrm{F}(7 ; 63)=9,20, \mathrm{p}<0,01]$ e prática aleatória-constante $[\mathrm{F}(7 ; 63)=6,38, \mathrm{p}<0,01]$.

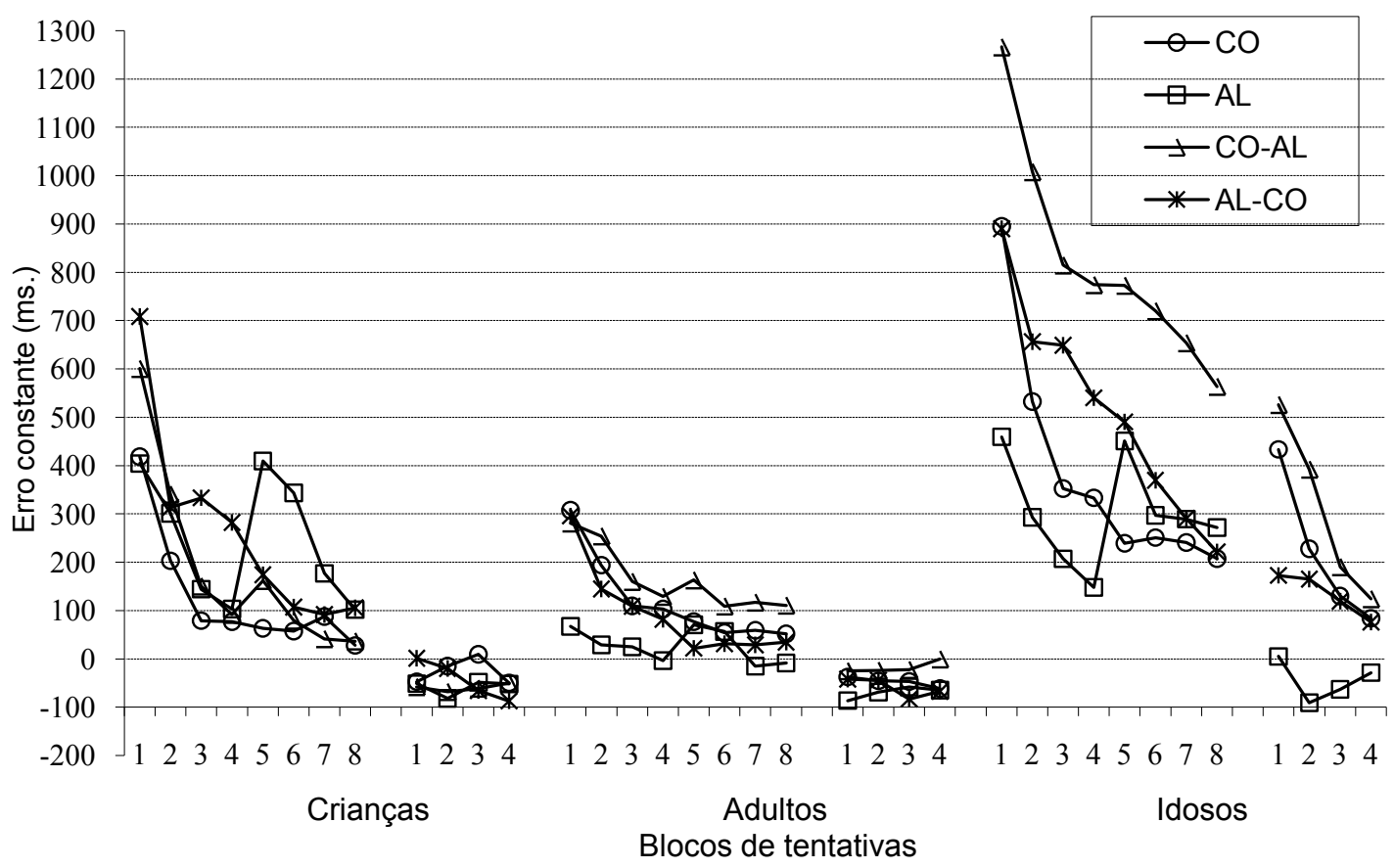

FIGURA 4 - Médias do erro constante em milissegundos, dos grupos de prática constante (CO), constante-aleatória (CO-AL), aleatória (AL) e aleatória-constante (AL-CO), das crianças, dos adultos e dos idosos, nos oito blocos de tentativas da fase de estabilização (1 a 8) e nos quatro blocos de tentativas da fase de adaptação (1 a 4).

Com relação aos adultos, verifica-se que, assim como ocorreu com as crianças, todos os grupos diminuíram o erro constante em direção à coincidência, ao longo da fase de estabilização. Essa observação foi confirmada pelo teste estatístico: prática constante $[\mathrm{F}(7 ; 77)=3,57, \mathrm{p}<0,01]$, prática constante-aleatória $[\mathrm{F}(7 ; 70)=3,7, \mathrm{p}<0,05]$, prática aleatória $[\mathrm{F}(7 ; 77)$ $=3,9, \mathrm{p}<0,01]$ e prática aleatória-constante $[\mathrm{F}(7$; 77) $=5,9, \mathrm{p}<0,01]$.

E, concernente aos idosos, todos os grupos diminuíram o erro constante, melhorando o desempenho na direção da coincidência ao longo da fase de estabilização: prática constante $[\mathrm{F}(7 ; 91)=11,3$, $\mathrm{p}<0,01]$, prática constante-aleatória $[\mathrm{F}(7 ; 91)=3,7$, $\mathrm{p}<0,01]$, prática aleatória $[\mathrm{F}(7 ; 98)=7,3, \mathrm{p}<0,01] \mathrm{e}$ prática aleatória-constante $[\mathrm{F}(7 ; 91)=11,0, \mathrm{p}<0,01]$.

Fase de adaptação

A análise de variância a três fatores apontou diferenças para o fator idade $[\mathrm{F}(2 ; 132)=9,02, \mathrm{p}<$ $0,00]$, para o fator blocos de tentativas $[\mathrm{F}(4 ; 528)$ 
$=19,20, \mathrm{p}<0,00]$, para a interação entre idade e blocos de tentativas $[\mathrm{F}(8 ; 528)=4,36, \mathrm{p}<0,00] \mathrm{e}$, para a interação entre idade, estrutura de prática e blocos de tentativas $[\mathrm{F}(24 ; 528)=1,69, \mathrm{p}<0,02]$. Não foram detectadas diferenças significantes para o fator estrutura de prática $[\mathrm{F}(3 ; 132)=1,20, \mathrm{p}$ $>0,30]$; para a interação entre idade e estrutura de prática $[\mathrm{F}(6 ; 132)=0,88, \mathrm{p}>0,50]$; e para a interação entre estrutura de prática e blocos de tentativas $[\mathrm{F}(12 ; 528)=1,55, \mathrm{p}=0,10]$.

\section{Discussão}

O objetivo desse estudo foi investigar os efeitos de diferentes estruturas de prática no processo adaptativo da aprendizagem de uma tarefa de "timing" coincidente em função da idade. A hipótese levantada foi que a prática constante-aleatória proporcionaria uma melhor adaptação comparada às práticas constante, aleatória e aleatória-constante, para crianças, adultos e idosos.

Em primeiro lugar, considerando que a pesquisa foi realizada em uma perspectiva de processo adaptativo de aprendizagem motora, os seguintes pressupostos devem ser destacados: o primeiro é de que na primeira fase deve ocorrer estabilização funcional; e, o segundo é de que os grupos sejam perturbados com a inserção da nova tarefa.

Neste sentido, verificou-se que na fase de estabilização: todos os grupos de prática constante, constantealeatória, aleatória e aleatória-constante de crianças, adultos e idosos melhoraram o seu desempenho em termos de precisão; com exceção do grupo de prática aleatória dos idosos, os grupos se tornaram mais consistentes; o desempenho de todos os grupos se tornou menos atrasado ao longo da fase; e, por fim, todos os grupos de crianças diminuíram a quantidade de execuções erradas, enquanto que nos grupos de adultos, apenas o grupo de prática aleatória-constante reduziu a quantidade e erros e, nos grupos de idosos, os grupos de prática aleatória e aleatória-constante apresentaram redução na quantidade de erros de execução.

Esse conjunto de resultados foi semelhante àqueles encontrados por CORRÊA (2001) no experimento em que a prática foi variada nos aspectos perceptivos e motores da tarefa. $\mathrm{O}$ fato de todos os grupos melhorarem, de modo geral, o seu desempenho significa que eles alcançaram uma estabilização funcional, ou seja, os movimentos foram se tornando padronizados e precisos ao longo da fase, permitindo inferir que houve a formação de uma estrutura. É
Nessa fase, os idosos apresentaram desempenho atrasado enquanto que os adultos e as crianças o fizeram de modo antecipado. Verificou-se, também, que os grupos apresentaram diminuição do erro constante, sendo que isso foi mais evidente para os grupos de idosos. E, que para esses últimos, o grupo de prática constante-aleatória foi aquele com menor erro. Além disso, observou-se que a prática constante-aleatória foi a única que em todas as idades, apresentou resposta adiantada em todos os blocos de tentativas.

importante lembrar que esse é um processo essencial e precedente àquele de adaptação (CORRÊA \& TANI, 2005; TANi, 1995; UGRINOWITSCH, 2003).

Conforme citado anteriormente, para poder observar o efeito de diferentes estruturas de prática no processo adaptativo, era fundamental, também, que a mudança da tarefa perturbasse o desempenho dos grupos. A fim de se garantir essa perturbação e, baseando-se em pesquisas anteriores (CORRÊA, 2001; TANI, 1995; UGRINOWITSCH, 2003), a tarefa foi modificada em aspectos perceptivo e motor. E, conforme esperado, observou-se piora do desempenho no fator blocos em termos de precisão e de consistência, permitindo inferir que, no geral, os grupos foram perturbados.

Com relação à fase de adaptação, a análise dos resultados mostrou que, na comparação entre grupos, a prática constante-aleatória proporcionou melhor desempenho no início da fase de adaptação do que a prática aleatória, em termos de precisão. Mostrou também que não houve diferenças significantes entre os grupos em nenhum bloco de tentativas da fase de adaptação tanto em termos de consistência e direção do desempenho, quanto de quantidade de execuçôes erradas.

Nas comparações em cada grupo, pôde-se observar que os grupos de prática constante e aleatóriaconstante foram os únicos que apresentaram aumento de variabilidade na mudança da tarefa. Constatou-se, também, que o grupo de prática constante-aleatória dos idosos diminuiu o erro constante quando a nova tarefa foi inserida, mudando seu desempenho de atrasado para adiantado, enquanto que os demais grupos se mantiveram atrasados. Além disso, verificou-se que o grupo de prática aleatória, de criança e de idosos, aumentou a quantidade de execuçōes erradas no início da fase de adaptação.

Esses resultados possibilitam entender que embora tenha sido verificado diferentes desempenhos entre 
crianças, adultos e idosos, sua aprendizagem foi igualmente beneficiada pela prática constante-aleatória.

Com relação a essa última observação, podese afirmar, então, que a hipótese apresentada foi confirmada. Quando a tarefa foi modificada, ela foi mais precisa do que a prática aleatória, não piorou a consistência, como ocorreu com as práticas constante e aleatória-constante e, não apresentou aumento de execuçôes erradas como o que ocorreu com os grupos de prática aleatória.

Pode-se dizer que, apesar de crianças, adultos e idosos serem diferentes, os processos que envolvem a aprendizagem não sofrem influência dos efeitos das diferentes estruturas de prática no processo adaptativo da aprendizagem de tarefas de "timing" coincidente. Os resultados encontrados no presente estudo corroboram aqueles de CORRÊA (2001), CORRÊA et al. (2003) e BARros (2006). Dessa forma, com base nas explicações desses autores, pode ser dito que tanto para crianças, quanto adultos e idosos a aprendizagem é beneficiada com a prática constante, em primeiro lugar, em virtude de ela possibilitar a formação da estrutura da habilidade ou formação de padrão de interação entre os componentes da habilidade. E, com a prática aleatória posteriormente, em razão de a mesma possibilitar a diversificação da habilidade e, portanto, o aumento de recursos para a adaptação.

Conforme apontado por CORRÊA (2001), é interessante observar como estruturas de características tão contrárias, juntas podem promover a melhor adaptação, mas quando isoladas, o benefício dessas estruturas no processo adaptativo é menor.

Segundo TANI (1989), dar uma ênfase única à prática constante pode levar a uma perda de flexibilidade na estrutura da habilidade, dificultando a sua adaptação. Isso porque esse tipo de prática é caracterizado pela repetiçãao da informação, estabilidade, consistência, ordem e precisão (CORRÊA, 2001). E foi exatamente isso que os resultados permitiram constatar: os grupos de prática constante foram os únicos que, na mudança da tarefa (no primeiro bloco de tentativas da fase de adaptação), pioraram o desempenho tanto em termos de precisão e quanto de consistência. Além disso, ao verificar que o grupo de prática aleatória-constante também apresentou piora de consistência no início da fase de adaptação, levanta-se a ideia de que a prática constante inserida após a prática aleatória também torna a estrutura rígida, dificultando a adaptação a novas situaçôes.

Por outro lado, destaca-se que a prática aleatória foi aquela que possibilitou pior desempenho do que as práticas constante e aleatória-constante ao final da fase de estabilização. Embora esses resultados sejam esperados devido características da prática aleatória de incerteza, instabilidade, inconsistência, desordem e erro (CORRÊA, 2001), o desempenho na fase de adaptação não foi o melhor. Além do grupo de prática aleatória ser menos preciso do que o grupo de prática constante-aleatória, nos idosos, foi o grupo que apresentou o desempenho mais atrasado. Verificou-se, também, que com a modificação na tarefa, os grupos de prática aleatória de crianças e idosos foram aqueles que apresentaram aumento da quantidade de erros de execução. Entretanto, é importante destacar que os grupos de prática aleatória mostraram melhora de desempenho com relação ao erro absoluto durante a fase de adaptação, igualando-se aos demais grupos no decorrer da fase.

Com respeito aos desempenhos dos grupos das diferentes idades, verificou-se que os idosos apresentaram pior desempenho que crianças e adultos. Observou-se ainda diminuição da variabilidade no decorrer da fase de adaptação nas três idades. Porém, os adultos tiveram menor variabilidade do que as crianças, que apresentaram menor variabilidade do que os idosos. Além disso, com relação à direção da resposta, crianças e adultos tiveram um desempenho próximo da coincidência (desempenho meta) e mudaram o comportamento de atrasado, ao final da fase de estabilização, para antecipado, durante a fase de adaptação. Já os idosos apresentaram erro maior (mais afastado da coincidência) do que crianças e adultos, e tiveram respostas atrasadas durante toda a fase. E, no erro de execução, crianças e idosos foram perturbados quando a tarefa foi modificada, mas depois retomaram o desempenho apresentado no final da fase de estabilização.

A dificuldade dos idosos em processar a realização o padrão motor solicitado de forma que coincidisse com o estímulo luminoso foi percebida durante a coleta, e confirmada nos resultados. Observou-se que muitos se preocuparam mais em realizar a sequência de forma correta do que em tentar "chegar" junto com a luz. Isso fica evidente, principalmente no grupo de prática aleatória que, apesar de apresentar menor precisão e desempenho mais atrasado, a quantidade de execuções erradas foi igual aos demais grupos. Interessante que alguns diziam, inclusive, que se a luz fosse retirada, ou que não tivessem que olhar para a canaleta no início da tarefa, eles seriam capazes de realizar as sequências com maior velocidade.

Segundo FerRaz (1993), o desempenho de tarefas complexas envolve boa capacidade de coletar e processar informaçóes que, por sua vez, permite que 
o indivíduo não precise monitorar a todo o momento a execução do movimento via "feedback" negativo, deixando o sistema livre para se preocupar com outros aspectos. Dessa forma, pode-se pensar que as perdas de certas capacidades tenham dificultado a formação da estrutura da habilidade e, por conseguinte, em os idosos terem apresentado menor consistência do desempenho e direcionamento da atenção mais para a execução da sequência de toques do que para os ajustes dela à velocidade do estímulo. Segundo SAntos, CorrêA e FREUDENHEIM (2003), as mudanças que ocorrem nessa idade podem afetar processos perceptivos, cognitivos, motores e de memória (fundamentais para a tarefa de "timing" coincidente) podem conduzir a perda de desempenho e, como se pôde verificar, a maior dificuldade na aprendizagem do que nas demais idades.

Apesar de ser o pior desempenho, verificou-se que apenas os idosos melhoraram a precisão durante a fase de adaptação e conseguiram, desse modo, se igualar ao desempenho das crianças ao final dessa fase. Esse resultado permite inferir que idosos têm apresentam capacidade de adaptação e, portanto, de aprendizagem, embora ela seja menor do que aquelas de adultos e crianças. Segundo Manoel (1994), é esperado que a eficiência diminua no envelhecimento, mas nem por isso a execução de uma habilidade motora deixará de ser competente. Isso porque, provavelmente, os planos de ação continuam a se modificar permitindo um ajuste ao que é possível executar, ou seja, possibilitando a adaptação a novas situaçôes.

Neste caso, cabe reforçar sobre os benefícios que uma prática de atividade física pode proporcionar a pessoas dessa idade, pois por mais que apresentassem dificuldades, melhoraram com a prática, foram capazes de alcançar bons níveis de desempenho. Essa capacidade de adaptação a novas tarefas proporcionadas pela prática já havia sido reportada por SANTOS e TANi (1995) no sentido de a prática de atividades que estimular as diversas capacidades e proporcionar a aprendizagem de novas habilidades. Eles sugeriram que a prática, no mínimo, retardaria a velocidade de perdas naturais da idade. Evidências nessa direção foram obtidas por Lobjois, BENGUigui e BERTSCH (2006) que mostraram superior desempenho numa tarefa de "timing" coincidente dos indivíduos que praticavam tênis comparados àqueles que não participavam de nenhum tipo de atividade física.

Com relação ao desempenho das crianças, os resultados corroboraram aqueles apresentados por FLEURY $\mathrm{e}$ BARD (1985) e se aproxima daqueles obtidos por FERRAZ (1993), uma vez que o erro absoluto foi semelhante àqueles dos adultos. De acordo com esses autores, a partir dos 11 anos (média da idade das crianças desse estudo), ocorre uma estabilização do desempenho com relação à precisão. Porém, nessa idade, a consistência do desempenho ainda é menor do que a dos adultos.

No que se refere aos adultos, conforme esperado observou-se os melhores desempenhos. Vale destacar que na mudança da tarefa, ou seja, no início da fase de adaptação, os adultos mantiveram a precisão, a consistência, bem como a quantidade de erros de execução. Por um lado, poderia ser pensado que a tarefa foi fácil, mas, por outro lado, ao observar que os adultos apresentaram erros absoluto e variável médio na fase de adaptação por volta de $100 \mathrm{~ms}$, verifica-se que, além da alta variabilidade, seu desempenho ficou longe da meta da tarefa (erro de até $40 \mathrm{~ms}$ ).

Finalizando, a consideração do objetivo e dos resultados do presente trabalho, pode-se concluir que o processo adaptativo na aprendizagem de crianças, adultos e idosos de uma tarefa de "timing" coincidente foi beneficiado pela prática constantealeatória. Por fim, para futuros estudos as seguintes perguntas são postas: esses resultados seriam replicados na aprendizagem de tarefas com outros tipos de exigência? Sabendo-se que a prática de atividades físicas influencia do desempenho, será que o efeito das diferentes estruturas de prática no processo adaptativo de aprendizagem motora também seria influenciado pelo nível e/ou tipo de atividade?

\section{Abstract}

Practice schedule and age on the adaptive process of the coincident timing task learning

The objective of this study was to investigate the effects of different practice schedules on the adaptive process of a coincident timing task learning in function of age. Children $(n=40)$, adults $(n=47)$ and elderly ( $n$ $=57$ ) were distributed into constant, random, constant-random and random-constant practice groups. The task consisted of touching response keys sequentially in conjunction with a visual stimulus. The experimental design was consisted of two learning phases: stabilization and adaptation. The data were analyzed in terms of 
absolute, variable, constant and execution errors. The results showed that the constant-random practice was the most efficient for the adaptive process in learning a coincident timing task regardless of the age group.

UnIterms: Practice schedule; Age; Coincident timing task; Adaptive process; Motor learning.

\section{Referências}

BARD, C.; FLEURY, M.; GAGNON, M. Coincidence-Antecipation timing: an age-related perspective. In: BARD, C; FLEURY, M.; HAY, L. Development of eye-hand coordination a cross the life spam. Columbia: University of South Carolina, 1990. p.283-305.

BARREIROS, J.M.P. Variabilidade das condiçōes de prática e interferência contextual: revisão de estudos. Lisboa: Ediçōes FMH, 1992.

. Variabilidade das condições de prática e interferência contextual em crianças e adultos. In: BARREIROS, J.M.P (Ed.).

O efeito de interferência contextual: contributos para o estudo da variabilidade do contexto de aprendizagem. Lisboa: Faculdade de Motricidade Humana, 1994.

BARROS, J.A.C. Estrutura de prática e processo adaptativo em aprendizagem motora. 2006. 131 f. Dissertação (Mestrado) - Escola de Educação Física e Esporte, Universidade de São Paulo, São Paulo, 2006.

CHOSHI, K. Aprendizagem motora como um problema mal-definido. Revista Paulista de Educação Física, São Paulo, p.17-23, 2000. Suplemento n.3.

CORRÊA, U.C. Estrutura da prática e processo adaptativo na aquisição de habilidades motoras. $2001,220 \mathrm{f}$. Tese (Doutorado) - Escola de Educação Física e Esporte, Universidade de São Paulo, São Paulo, 2001.

Prática constante-variada e a aquisição de habilidades motoras. 2007. 109 f. Tese (Livre Docência) - Escola de Educação Física e Esporte, Universidade de São Paulo, São Paulo, 2007.

CORRÊA, U.C.; BARROS, J.A.C.; MASSIGLI, M.; GONÇALVES, L.A.; TANI, G. A prática constante-aleatória e o processo adaptativo de aprendizagem motora: efeitos da quantidade de prática constante. Revista Brasileira de Educaçáo Física e Esporte, São Paulo, v.21, n.4, p.301-14, 2007.

CORRÊA, U.C.; BENDA, R.N.; MEIRA JÚNIOR, C.M.; TANI, G. Practice schedule and adaptative process in the acquisition of a manual force control task. Journal of Human Movement Studies, London, v. 44, p.121-38, 2003.

CORRÊA, U.C.; BENDA, R.N.; TANI, G. Estrutura de prática e processo adaptativo na aquisição do arremesso de dardo de salão. Revista Brasileira de Ciências do Esporte, Campinas, v.22, n.2, p.69-83, 2001.

CORRÊA, U.C.; GONÇALVES, L.A.; BARROS, J.A.C.; MASSIGLI, M. Prática constante-aleatória e aprendizagem motora: efeitos da quantidade de prática constante e da manipulação de exigências da tarefa. Brazilian Journal of Motor Behavior, Rio Claro, v.1, p.41-52, 2006.

CORRÊA, U.C.; OLIVEIRA, P.H.V.; OLIVEIRA, J.A.; FREUDENHEIM, A.M.; PAROLI, R.; UGRINOSWITSCH, H.; MEIRA JÚNIOR, C.M.; MARINOVIC, W.; SIMONI, C.G.; TANI, G. "Timing” coincidente em tarefas complexas: estudo exploratório do desempenho de adultos de diferentes idades em diferentes velocidades de estímulo visual. Revista Brasileira de Educação Física e Esporte, São Paulo, v.19, n.4, p.301-15, 2005.

CORRÊA, U.C.; TANI, G. Aparelho de timing coincidente em tarefas complexas. PI no 0.4.04.433-4 de 03/08/2004. Revista da Propriedade Industrial, n.1763, p.178, 19/10/2004.

. Estrutura de prática e processo adaptativo em aprendizagem motora: por uma nova abordagem da prática. In: TANI, G. (Ed.). Comportamento motor: aprendizagem e desenvolvimento. Rio de Janeiro: Guanabara Koogan, 2005. p.141-61. DOUVIS, J.S. Variable practice in learning the forehand drive in tennis. Perceptual and Motor Skilss, Missoula, v.101, p.531-45, 2005.

FERRAZ, O.L. Desenvolvimento da capacidade de timing antecipatório em crianças. 1993. 100 f. Dissertação (Mestrado) - Escola de Educação Física e Esporte, Universidade de São Paulo, São Paulo, 1993.

FIALHO, J.V.A.P. A prática constante-aleatória: efeitos da manipulação do aspecto da habilidade e o tipo de perturbação no processo adaptativo em aprendizagem motora. 2007. 295 f. Dissertação (Mestrado) - Escola de Educação Física, Fisioterapia e Terapia Ocupacional, Universidade Federal de Minas Gerais Belo Horizonte, 2007.

FLEURY, M.; BARD, C. Age, stimulus velocity and task complexitu as determiners of coincident timing behavior. Journal of Human Movement Studies, London, v.11, p.305-17, 1985. 
LOBJOIS, R.; BENGUIGUI, N.; BERTSCH, J. The effect of aging and tennis playing on coincidence-timiming accuracy. Journal of Aging and Physical Activity, Champaign, v.14, p.75-98, 2006.

MANOEL, E.J. Desenvolvimento motor: implicações para a educação física escolar I. Revista Paulista de Educação Física, São Paulo, v.8, n.1, p.82-97, 1994.

MASSIGLI, M.; GONÇALVES, L.A.; BARROS, J.A.C.; SOUZA JÚNIOR; CORRÊA, U.C. O efeito de diferentes quantidades de prática constante-aleatória no processo adaptativo na aquisição de habilidades motoras: manipulação de exigências perceptivas da tarefa. In: CONGRESSO INTERNACIONAL DE EDUCAÇÃO FÍSICA: FIEP 2004; CONGRESSO DE FISIOTERAPIA DO MERCOSUL E CONGRESSO CINETÍFICO DA FIEP, 19., 2004, Foz do Iguaçu. Anais... Foz do Iguaçu, FIEP, 2004. p.131.

PAROLI, R. Efeito da estrutura de prática na aquisição de uma habilidade motora. 2004. Dissertação (Mestrado) - Escola de Educação Física e Esporte, Universidade de São Paulo, São Paulo, 2004.

PINHEIRO, J.P.; CORREAA, U.C. Estrutura de prática na aquisição de uma tarefa de timing coincidente com desaceleração do estímulo visual. Revista Portuguesa de Ciências do Desporto, Porto, v.7, n.3, p.0-0, 2007.

POLLOCK, B.J.; LEE, T.D. Dissociated contextual interference effects in children and adults. Perceptual and Motor Skills, Missoula, v.84, p.851-58, 1997.

SANTOS, S.; CORRÊA, U.C.; FREUDNHEIM, A.M. Variabilidade de performance numa tarefa de "timing” antecipatório em indivíduos de diferentes faixas etárias. Revista Paulista de Educação Física, São Paulo, v.17, n.2, p.154-62, 2003.

SANTOS, S.; TANI, G. Tempo de reação e a aprendizagem de uma tarefa de "timing" antecipatório em idosos. Revista Paulista de Educação Física, São Paulo, v.9, n.1, p.51-62, 1995.

SHEA, C.H.; PARK, J.H.; BRADEN, H.W. Age-related effects in sequential motor learning. Physical Therapy, Alexandria, v.86, n.4, p.478-88, 2006.

SILVA, J.A.O.; ARAÚJO, U.O.; TANI, G.; CORREAA, U.C. Estrutura de prática e complexidade da tarefa no processo adaptativo de aprendizagem motora. Revista da Educação Física/UEM, Maringá, v.20, n.3, p.313-23, 2009.

TANI, G. Variabilidade de resposta e processo adaptativo em aprendizagem motora. 1989. Tese (Livre docência) - Escola de Educação Física, Universidade de São Paulo, São Paulo, 1989.

Organização hierárquica do comportamento motor humano. São Paulo: [s.n.], 1995. (Relatório Final de atividades de Pós-doutorado, University of Sheffield).

. Processo adaptativo: uma concepção de aprendizagem motora além da estabilização. In: TANI, G. (Ed.). Comportamento motor: aprendizagem e desenvolvimento. Rio de Janeiro: Guanabara Koogan, 2005. p.60-70.

TERTULIANO, I.W. Estrutura de prática e frequiência de feedback extrínseco na aprendizagem de habilidades motoras. 2007. 71 f. Dissertação (Mestrado) - Escola de Educação Física e Esporte, Universidade de São Paulo, São Paulo, 2007. UGRINOWITSCH, H. Efeito do nível de estabilização e do tipo de perturbação no processo adaptativo em aprendizagem motora. 2003. Tese (Doutorado) - Escola de Educação Física e Esporte, Universidade de São Paulo, São Paulo, 2003. WALTER, C. Estrutura de prática e liberdade de escolha na aprendizagem de habilidades motoras. 2007. $96 \mathrm{f}$. Dissertação (Mestrado) - Escola de Educação Física e Esporte, Universidade de São Paulo, São Paulo, 2007.

ENDEREÇO

Lucia Afonso Gonçalves

Escola de Educação Física e Esporte - USP

Av. Prof. Mello Moraes, 65

05508-030 - São Paulo - SP - BRASIL e-mail: lugaf_sp@yahoo.com.br
Recebido para publicação: 27/05/2009

Revisado: 15/12/2009

Aceito: 10/03/2010 